\title{
The study of ethnic attitudes during interactions with avatars in virtual environments
}

\author{
Galina Ya. Menshikova*, Olga A. Saveleva, Yury P. Zinchenko \\ Faculty of Psychology of Lomonosov Moscow State University, Moscow, Russia \\ *Corresponding author. E-mail: gmenshikova@gmail.com
}

Background. Modern technologies provide a wide range of opportunities for studying different types of social processes and phenomena. Currently many original social studies have been done with the use of virtual reality technologies. The effectiveness of their application has been shown for the study of verbal and nonverbal communication; the processes of ethno-cultural identity; and for teaching social skills, as well as correcting social anxiety and ethnic attitudes. One of the very real question concerning spatial behavior during communication with partners from other ethnic groups, however, has not been studied very much.

Objective. In our study we explored proxemic behavior in subjects' face-to-face interactions with avatars of in-group and out-group ethnic appearance. Using the CAVE virtual reality system, we studied preferred interpersonal distances in carrying out memory tasks during interaction with the avatars.

Design. Three virtual environments with avatars of different ethnic appearance were developed. Each virtual scene represented a room where three avatars of the same ethnicity were standing. Their appearance was associable with one of three ethnic groupsthe Slavic, North Caucasian, or the Central Asian. The participants (all of whom identified themselves as Russians) were immersed in the virtual scenes with the help of the CAVE virtual reality system. They were instructed to keep in mind as many details of the avatars' appearance as they could.

During the task's execution the interpersonal distances between the participants and the avatars were registered. After leaving the CAVE, the participants were asked to answer questions about the details of avatars' appearance, and to fill out a questionnaire assessing the Presence Effect in virtual environments. The identification accuracy of the avatars' appearance details and the Presence effect were measured. The interpersonal distances were analyzed for the area around the direction of mutual gaze.

Results. The results showed that participants preferred to keep closer interpersonal distances from the avatars of the same ethnic group as their own. During interaction with avatars belonging to another ethnic group, significantly larger interpersonal distances were preferred. A significant correlation between the interpersonal distance and the Presence Effect was also revealed. 
Conclusion. Virtual reality technology provides a unique and valuable tool for social researchers, including in ethnic attitude studies. A complex method of measuring interpersonal distances and the Presence Effect allows us to assess the main variables during social interaction with high accuracy. The virtual environments designed for this study can be applied successfully not only for studying proxemic behavior, but also for accomplishing other tasks, such as developing communication skills and forming positive attitudes towards ethnic out-groups.

Keywords: interethnic attitudes, proxemics, nonverbal communication, compensation effect, mutual gaze, CAVE virtual reality technology, avatar, Presence Effect

\section{Introduction}

Social studies are currently getting more efficient due to modern technologies, which allow us to design complicated stimuli scenes and detect behavioral reactions in real time. Virtual reality systems, which have been increasingly used for these studies during the past 20 years, are one example of such technologies. One of the main advantages of using virtual reality systems in socialstudies is that they provide an opportunity to study the social interaction between the study's participants and virtual characters, i.e. avatars (Lanier, 1992; Bailenson at al., 2008a; Eichenberg, 2012). According to the Oxford dictionary, the term „avatar" is defined as , an icon or figure representing a particular person in a video game, Internet forum, etc." (www.oxforddictionaries.com/definition/ english/avatar). In the context of our study, we considered avatars to be virtual partners simulating real people's appearance and behavior during interaction in virtual environments.

The active application of virtual reality systems to socialstudies allows researchers to extend the range of fundamental and applied research objectives which, until recently, could hardly (if ever) be accomplished using classical methods such as questionnaires, surveys, etc. Thus, during the past decade, study of ethno-cultural identification, interethnic and interracial attitudes, and the nonverbal behavior of partners from different cultures has developed a lot (Blascovich et al. 2002; Asmolov et al., 2014; Zinchenko et al., 2015). New methods of analysis and for regulation of social conflicts have been designed due to the new tasks. Thereby, using avatars as virtual partners provides us with original data on the peculiarities of personal space characteristics (Bailenson et al., 2003), the factors determining spatial behavior in virtual environments (Bailenson et al., 2008b), and the specific manifestations of social preferences (McCall et al., 2009).

The dynamics of spatial behavior during communication are among the most significant expressions of interpersonal (and, especially, ethnic) attitudes. The study of spatial behavior is called proxemics (Hall, 1966). Different proxemic signals used in social contacts have been identified, including interpersonal distance, gaze direction, head and body posture, shoulder orientation, and others (Hall, 1966; Schegloff, 1998). The degree of interpersonal distance is considered to be one of the most expressive non-verbal signals in the communication process. A number of distance zones have been distinguished: "Intimate Space" (0.15-0.45 m); "Personal Space" (0.46-1.5 m); "Social Space" (1.6-3.7 m); and "Public Space" ( $\geq 3.7 \mathrm{~m})$. The proxemic zone values are assumed to reflect some implicit rules associated with social laws 
of secure communication: by observing proxemic rules, people share information about their opinions on social status, race/ethnicity, and cultural features.

The degree of spatial proximity between communicants has been shown to depend on many factors, including gender and age characteristics (Willis, 1966), social prejudices, and stereotypes (Watson, 1970), as well as racial/ethnic preferences (Rosegrant \& McCroskey, 1975). A compensation effect in interpersonal distances has been found, which shows that people maintain greater distances from a partner who maintains a constant mutual gaze (Argyle, Dean, 1965). That same effect was found in the interaction between the participants and the avatars (Bailenson et al., 2003). It was shown also that psychological discomfort arises in the case of personal space violation by another person, especially in the case that he/she has a different ethnic affiliation. This sense may manifest itself in involuntary behavioral reactions of contact avoidance: participants prefer to increase the interpersonal distance between themselves and a partner of a different racial/ethnic group.

Methods have been developed for studying the rules of proxemics (Hayduk, 1983), which include:

- The chair selection method, in which a participant himself chooses a place which he/she considers comfortable for communication;

- The stop distance method, in which a participant tells a partner to stop approaching, when the partner reaches the most comfortable distance for communication;

- The method of projective studies, in which participants manipulate puppets or abstract figures; and

- The natural-observation method, when the spatial behavior of communicants in real situations is recorded.

Analysis of the details of their application has revealed both the advantages and disadvantages of each method. Thus, the method of projective studies has been shown to have low ecological validity, while the natural observation method shows a low level of control over the scene's physical parameters. A significant drawback of the other methods is related to the need to involve actors whose behavior (e.g., gestures, facial expressions, voice timbre, etc.) can change a lot between experimental trials. Also, it should be noted that in studies of ethnic preferences, the main difficulty lies in the choice of actors, whose appearance should reflect the representative features of the ethnic groups being studied.

The use of virtual reality systems eliminates most of the above-mentioned disadvantages. Analysis of the application of virtual reality technology for studying social phenomena has shown its obvious benefits (Zinchenko et al., 2015; Fox et al., 2009). The main ones include the ecological validity of virtual environments, and the ability to registrate full real-time responses of the respondent's behavior (Zinchenko et al., 2010). Furthermore, it should be noted that the technical parameters of virtual reality systems provide high-accuracy estimations of the participants' involuntary reactions during communication with virtual partners.

In earlier proxemic studies, the evaluation of spatial metrics was quite rough, thereby limiting the potential for accurate quantitative analysis of the spatial behavior (Jones, Aiello 1979). The more recent development of head- and body-tracking technology in virtual environments enables researchers to record changes in macro and micro movements during communication with a high accuracy (Oosterhout 
\& Visser 2008; Gamberini et al. 2015). It is also important to mention one more advantage: the ability to standardize the situation of ethnic/racial interaction due to the ability to control the avatars' behavior (their facial expressions, postures, and etc.).

One of the most important issues in applying virtual environments to social studies is the question of how realistic and natural the virtual scenes and virtual partners appear to the participants. It is assumed that the more realistic the perception of a virtual environment, the stronger the feeling of immersion in the virtual world. Immersive experience also suggests the feeling of "being there," as well as one's own body's presence inside the virtual environments (Lombard \& Ditton, 1997).

To assess the feeling of immersion, B. Witmer and M. Singer suggested the "Presence Effect" concept. They defined it as "the subjective experience of being in one place or environment, even when one is physically situated in another" (Witmer \& Singer, 1998). A questionnaire was developed to elicit the participants' sense of "presence" in virtual environments, together with a questionnaire for measuring a person's immersive tendencies. It included three to four general, and five to six specific questions concerning the realism of the virtual scenes and characters. The questionnaire and its modifications have become a standard tool for measuring the efficiency of virtual reality exposure.

While virtual reality systems have obvious benefits for studying ethnic/race preferences, there are few publications on this issue. Among these works, we can distinguish the study (Dotsch \& Wigboldus, 2008) which showed that people with a negative attitude toward some racial group showed the same racial prejudices during interaction with avatars with a corresponding appearance. Virtual systems are successfully used to assess the social attitudes toward the Negroid race (McCall et al., 2009). Groom (Groom et al., 2009) discovered changes in the subjects' racial attitudes after they manipulated their own avatar by giving it a modified skin color.

The goal of our experiment was to study the spatial behavior (proxemics) in face-to-face interactions with avatars of in-group and out-group ethnic appearance. We hypothesized that the interpersonal distance would decrease during communication with avatars with an in-group ethnic appearance, and, conversely, it would increase in the case of interaction with avatars with an out-group ethnic appearance. We also assumed that the value of interpersonal distance would correlate with the strength of the Presence Effect.

\section{Method}

Subjects. 40 persons participated (29 female, 11 male), aged 18 to 26 . All participants identified themselves as Russians (belonging to the Slavic ethnic group). All of them had normal or corrected-to-normal vision and had no disorders of the vestibular system or brain injuries.

Stimuli. Three-dimensional virtual scenes simulating the situation of inter-ethnic interaction were developed. Each scene represented a living room in the center of which a group of three avatars of the same ethnic appearance were located. There were windows and a door in the room, with a hilly landscape visible through them. 
The avatars stood in a circle facing each other. They differed in height, build, clothing details, facial features, and facial expressions: neutral, less friendly, and friendlier. The avatars were animated: they swayed a little, moved eyes and heads. Three virtual scenes were developed: the first contained three avatars of Slavic appearance; the second, avatars of North Caucasian appearance; and the third, avatars of Central Asian appearance.

Apparatus. Virtual scenes were presented using the CAVE virtual reality system Barco Ispace 4 (Fig. 1). It consisted of four large flat screens, which were combined to form a cube consisting of three walls and a floor. The effect of three-dimensional virtual objects and characters was created using Crystal Eyes glasses. The participants used a Flystick 2 manipulator to navigate in the virtual environments. Position data of the participants' movements were recorded using tracking system A.R.T. DTrack 2. Position points were recorded with an $8 \mathrm{~Hz}$ update frequency. VirTools 4.0 was used for software development. This system has been successfully used in the research of three-dimensional illusions (Menshikova, 2013) and disorders of vestibular function (Menshikova et al., 2015).

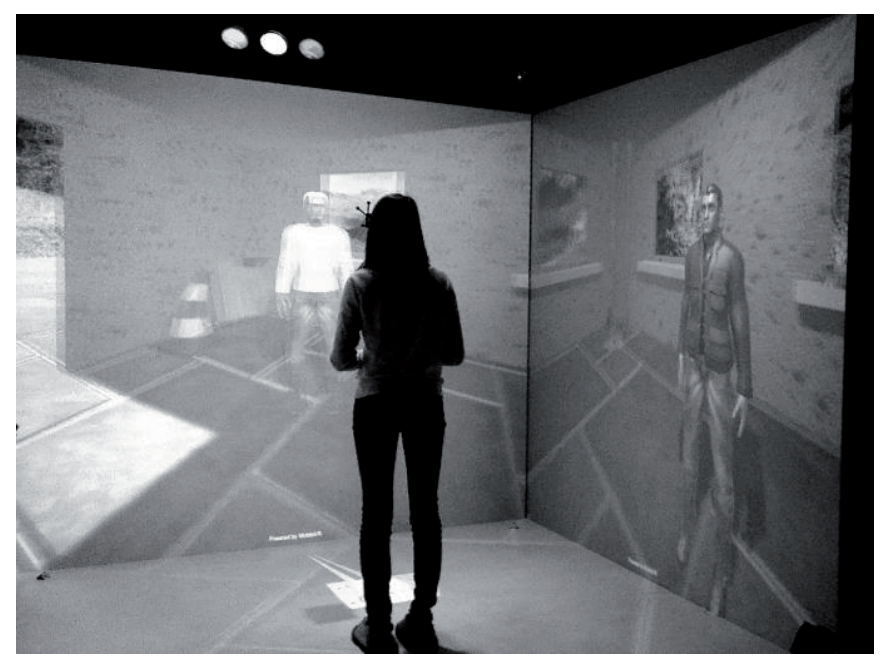

Figure 1. An overview of the CAVE virtual reality system

Procedure. A scenario for the participants' action in the virtual environment was developed. In the beginning, the participants were offered a training session, where they could learn about their possible movements in the virtual environment using Flystick. Each participant was asked to move around the room in all directions, approach the avatars, and get around them. The training session lasted for 5-10 minutes, during which time the participant had mastered the Flystick in order to fully control her/his own movement in the virtual environment.

Then she/he took the main test, which consisted of three sessions. In the first session, the participants interacted with a group of avatars of Slavic appearance; in the second, of North Caucasian appearance; in the third, of Central Asian appearance. The sequence of presentation of the different ethnic groups was randomized.

Before the testing, the following instruction was given: "You are participating in an experiment assessing your accuracy in recollecting the appearance of various 
avatars. Three scenes representing a room with three avatars are presented. Your task is to approach each avatar using Flystick and remember all the details of its appearance (i.e. clothing details, facial features, facial expression). Then you have to fill out two questionnaires concerning the details of each avatar's appearance, as well as your ethnic preferences toward the avatars you have just seen. Then the second and third scenes will be presented, and the testing of the accuracy of your recollection will be repeated. At the end you will be asked to complete a questionnaire concerning the realism of virtual scenes."

The questionnaire assessing the recognition accuracy of the avatars' appearance was designed for each ethnic group separately. It consisted of eight questions about the details of clothing, eye and hair color, the peculiarities of emotional facial expressions, and others.

The questionnaire for assessing the Presence Effect was a modified version of the Witmer \& Singer Questionnaire (Witmer \& Singer, 1998), translated by one of the authors (G. Menshikova), and modified in accordance with the objectives of our study. It consisted of 10 questions relating to the general impressions of the virtual environments, the realism of their appearance, and the behavior of the avatars.

To be specific: Three general questions were formulated about the realism of the virtual environments (numbers 1-3); two questions about the realistic properties of the avatars' appearance (numbers 4,5); three questions about the feelings caused by interaction with the avatars (numbers 6-8); and two questions about the participant's adaptation to the virtual environment (numbers 9,10). The participants evaluated the questions on a scale from 1 to 7 , where one corresponded to minimum, and seven to maximum virtual environment effect.

The variables to be measured were the pathways of the participants' walking around avatars of different ethnic appearance than themselves; the execution time of any session; and the answers to the questionnaire assessing the recognition accuracy of the appearance of avatars from each ethnic group, as well as the answers to the questionnaire assessing the Presence Effect.

Data processing. The data analysis included the calculation of the average and minimal average distances that participants assumed between themselves and avatars of different ethnic groups. To reduce the scatter of data points, the average and minimum distances were summarized in a particular space area around the direction of mutual gaze. The choice of this area was driven by information showing that the participants prefer to maintain a greater distance while in the "eyeto-eye" area, and a shorter distance if they exit the eye contact zone (Bailenson et al., 2003). The strength of the Presence Effect was calculated with scores averaged over the entire sample, and over each group of avatars. Data analysis was made by SPSS Statistics 20.

\section{Results and discussion}

The accuracy of the recognition of the avatars' appearance in different ethnic groups. The accuracy of the avatars' appearance recognition, and also the avatars' facial expression recognition, was calculated across the entire sample separately for each group of avatars. It was shown that the recognition accuracy of certain details remained consistently high regardless of the ethnicity of the group of avatars: $\mathrm{P} 1=$ 
$0.98 \pm 0.02 ; \mathrm{P} 2=0.97 \pm 0.015$; and $\mathrm{P} 3=0.98 \pm 0.01$, where $\mathrm{P} 1, \mathrm{P} 2$, and $\mathrm{P} 3$ were the probabilities of identifying the details of avatars of Slavic, North Caucasian and Central Asian appearance, respectively. A negligible decrease in the identification accuracy was recorded for fine details (e.g., the ring on a finger), for details outside the range of active attention (e.g., shoe color), and also for the recognition of the avatars' facial expressions. For these cases, no significant probability difference across various avatar ethnic groups was observed as well. The results indicated the absence of a significant influence of the ethnicity factor (the situation of social interaction with avatars of different ethnic groups) on the execution of the cognitive memory task.

Inter-individual distance between a participant and the avatars of ethnic in-and out-groups. The specific characteristics of approaching and walking around avatars belonging to different ethnic groups, were mapped by registering the $\mathrm{X}$ and $\mathrm{Y}$ coordinates of the participant's location in the virtual environment. In Fig. 2, three pathways around the avatars of different ethnic appearance performed by a typical participant (A.G.) are shown. The avatars' 2D positions are marked with black circles (numbers 1, 2, 3); the starting point of the participant's position with a brown circle (number 4). The trajectories of the participants' bypass around avatars of different ethnic appearances are marked with different colors: green, red, and blue lines for the bypass around Slavic, North Caucasian, and Central Asian groups, respectively.

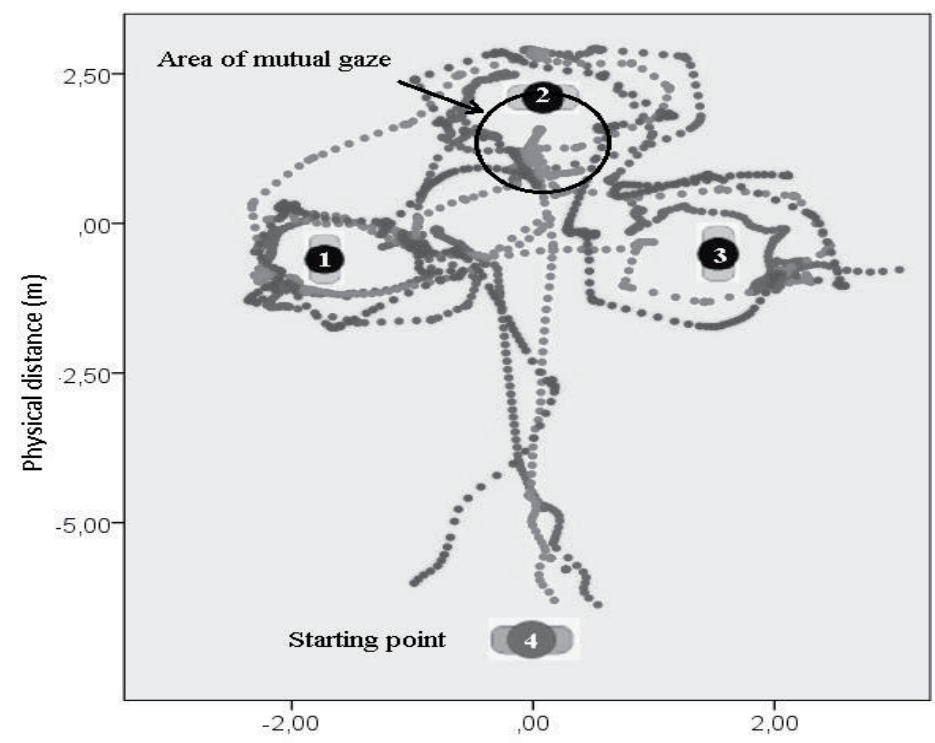

Figure 2. An example of the 3 pathways of a typical participant as she walks from the starting point around the avatars of different ethnic appearance (the red line=Slavic appearance; the green line $=$ North Caucasian appearance; the blue line $=$ Central Asian appearance)

The values of interpersonal distances varied strongly over the entire sample because the participants expressed their implicit attitudes toward the avatars by variable behavioral reactions. Each participant's motion trajectory around the ava- 
tars was analyzed, in order to reveal the impact of ethnic preference on the interpersonal distances. The average and minimum average distances for each ethnic avatar group were calculated. Distance estimates were made in a space limited to $\pm 30^{\circ}$ of the visual angle around the direction of mutual gaze. In Fig. 2 this area is marked with a black circle.

The average and minimum average interpersonal distances (in meters) for avatars of Slavic (Group 1), North Caucasian (Group 2), and Central Asian (Group 3) appearance can be seen in Table 1. Standard deviations are shown in brackets.

Table 1. Interpersonal distance: Descriptive statistics

\begin{tabular}{|c|c|c|c|}
\hline Interpersonal distance & Group 1 & Group 2 & Group 3 \\
\hline Average distance $(\mathrm{m})$ & $1.16(0.16)$ & $1.20(0.14)$ & $1.21(0.15)$ \\
\hline Minimum average distance $(\mathrm{m})$ & $0.57(0.14)$ & $0.68(0.19)$ & $0.73(0.18)$ \\
\hline
\end{tabular}

In Fig. 3 the values of average (dark bars) and minimum average (light bars) interpersonal distances are shown. Their values are presented separately for the avatars of different ethnic groups: the Slavic (Group 1), the North Caucasian (Group 2), and the Central Asian (Group 3).

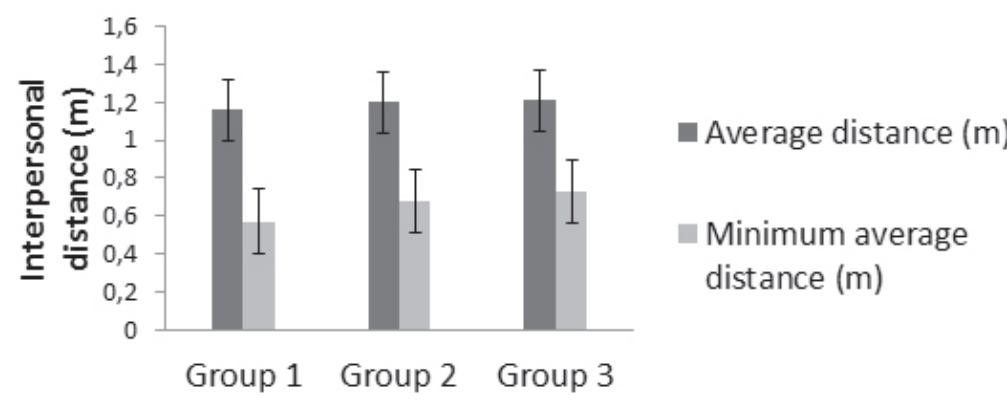

Figure 3. The average (dark bars) and minimum average (light bars) interpersonal distances for avatars of the Slavic (Group 1), North Caucasian (Group 2), and Central Asian (Group 3) groups

The differences between the average interpersonal distances with pairwise comparison of all groups were insignificant: between Groups 1 and $2(\mathrm{t}(80)=3.67$, $\mathrm{p}<0.08)$; between Groups 1 and $3(\mathrm{t}(80)=4.65, \mathrm{p}<0.09)$; and in Groups 2 and 3 $(\mathrm{t}(80)=5.44, \mathrm{p}<0.13)$. When comparing the minimum distances, we found that the differences between Groups 1 and 2, as well as between Groups 1 and 3, were significant: $\mathrm{t}(80)=2.48, \mathrm{p}<0.05)$ and $\mathrm{t}(80)=3.86, \mathrm{p}<0.04)$, respectively. On the contrary, the differences of minimum distances between Groups 2 and 3 were insignificant $(\mathrm{t}(80)=5.44, \mathrm{p}<0.13)$.

Thus, the minimum distance data confirmed our hypothesis: its decrease during communication with avatars of in-group ethnic appearance, and, conversely, its increase in the case of interaction with avatars of out-group ethnic appearance. It should be mentioned that the values of minimum distances observed in our ex- 
periment $(0.58 \mathrm{~m}-0.72 \mathrm{~m})$ were higher than the findings $(0.34 \mathrm{~m}-0.54 \mathrm{~m})$ of a similar study performed with the help of virtual reality systems (Bailenson et al., 2003). Perhaps the decrease in the minimum distances was due to the particular memory tasks given to the participants: in the Bailenson study, the participants were asked to remember the avatars' names, which were written on their clothing in small letters, and thus required maintaining shorter distances from the avatars.

Measuring the Presence Effect. Presence scores were averaged separately for each question over all participants and all avatars' ethnic groups. The mean Presence scores for each question are shown in Fig. 4.

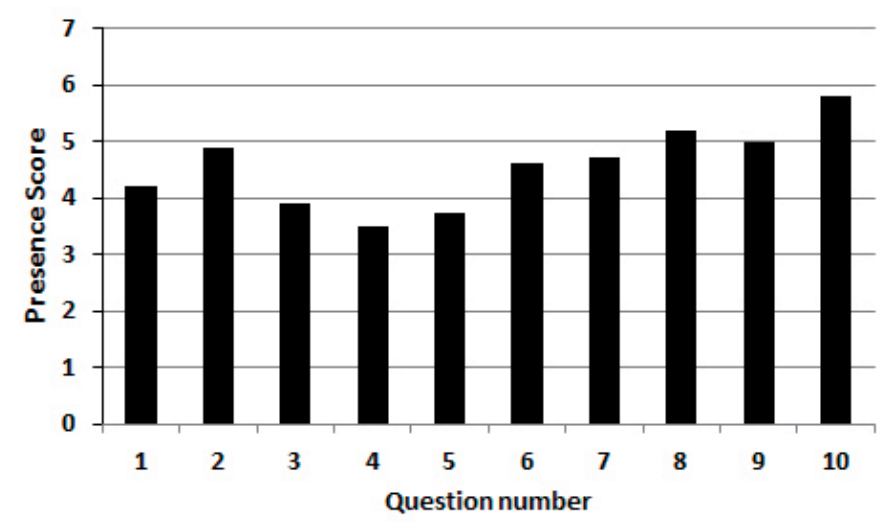

Figure 4. Mean Presence scores versus the question number

Our data showed that the highest scores ( $>5$ points) were given in response to predictions of further events in a virtual environment, as well as in the estimates of the speed of adaptation to a virtual environment. The least pronounced Presence Effect ( 3.5-4 points) was shown for the evaluation of the avatars' realism and the naturalness of their movements. The answers to the remaining questions about the realism of the virtual environments were in the middle range of 4-5 points.

We calculated the sample correlation coefficient between the values of mean minimum interpersonal distances and Presence scores, averaged over all 10 questions. The results showed a high negative correlation $(\mathrm{r}=0.67 ; \mathrm{p}=0.005)$, indicating a more pronounced interpersonal effect if the participant's Presence score was higher. In other words, the more realistic the virtual environment is perceived to be, the larger distances the participants kept from the avatars, regardless of their ethnic appearance.

The question arises whether the same behavior would be observed when the participants interact with real people in a real environment. We assume that our results are valid and can describe the real spatial behavior during interaction with partners of other ethnic groups. This assumption is based on the following ideas. First, it has been shown that many social phenomena manifest themselves in the same way in both real and virtual environments (Bailenson et al., 2003; Fox et al., 2009; Eichenberg, 2012). Second, the similarity of the impact of both environments (real and virtual) on social behavior is indirectly confirmed by the high scores of the Presence Effect. Third, our participants described the avatars as real people in the interview session at the end of the experiment. 


\section{Conclusion}

Virtual reality technology provides a unique and valuable tool for social research, including ethnic attitude studies.

The assessment of interpersonal distances maintained between participants and avatars of different ethnic appearance, as well as the evaluation of the Presence Effect, allow us to measure ethnic attitudes.

Our method allows us to control and assess a large number of variables during social interaction, including an estimation of proxemics, with high accuracy.

Our method showed that respondents preferred to keep shorter interpersonal distances from avatars of the same ethnic appearance as themselves. During the interaction with avatars with out-group ethnic appearance, significantly larger interpersonal distances were preferred.

These results could be of great importance for developing complex methods of testing behavioral patterns during social interactions.

The virtual environments we designed can be applied successfully not only to studying the respondents' ethnic attitudes, but also to solving other practical tasks, such as developing communication skills with, and forming positive attitudes toward, ethnic out-groups.

\section{Acknowledgements}

The research was supported by the Russian Science Foundation (project No 1518-00109) and used equipment purchased by the Program of Development of the Lomonosov Moscow State University to 2020.

\section{References}

Argyle, M., \& Dean, J. (1965). Eye-contact, distance and affiliation. Sociometry, 28(3), 289-304. doi: $10.2307 / 2786027$

Asmolov, A. G., Shekhter, E. D., \& Chernorizov, A. M. (2014). Beyond homeostasis: an historical and evolutionary approach to the development of complex systems [In Russian: Po tu storonu gomeostaza: istoriko-evolyutsionnyj podhod k razvitiyu slozhnyh system]. Voprosy psihologii, 4, 3-13.

Bailenson, J. N., Yee, N., Blascovich, J., \& Guadagno, R. E. (2008a). Transformed social interaction in mediated interpersonal communication. Mediated interpersonal communication, 6 , 77-99.

Bailenson, J., Patel, K., Nielsen, A., Bajscy, R., Jung, S. H., \& Kurillo, G. (2008b). The effect of interactivity on learning physical actions in virtual reality. Media Psychology, 11(3), 354-376. doi: $10.1080 / 15213260802285214$

Bailenson, J. N., Blascovich, J., Beall, A. C., \& Loomis, J. M. (2003) Interpersonal distance in immersive virtual environments. Personality and Social Psychology Bulletin, 29, 1-15. doi: $10.1177 / 0146167203029007002$

Blascovich, J., Loomis, J., Beall, A. C., Swinth, K. R., Hoyt, C. L., \& Bailenson, J. N. (2002). Immersive virtual environment technology as a methodological tool for social psychology. Psychological Inquiry, 13(2), 103-124. doi: 10.1207/S15327965PLI1302_01

Dotsch, R., \& Wigboldus, D. H. (2008). Virtual prejudice. Journal of Experimental Social Psychology, 44(4), 1194-1198. doi: 10.1016/j.jesp.2008.03.003 
Eichenberg, C. (2012). Virtual reality in psychological, medical and pedagogical applications. doi: $10.5772 / 2607$

Fox, J., Arena, D., \& Bailenson, J. N. (2009). Virtual reality: A survival guide for the social scientist. Journal of Media Psychology, 21(3), 95-113. doi: 10.1027/1864-1105.21.3.95

Gamberini, L., Chittaro, L., Spagnolli, A., \& Carlesso, C. (2015). Psychological response to an emergency in virtual reality: Effects of victim ethnicity and emergency type on helping behavior and navigation. Computers in Human Behavior, 48, 104-113. doi: 10.1016/j. chb.2015.01.040

Groom, V., Bailenson, J. N., \& Nass, C. (2009). The influence of racial embodiment on racial bias in immersive virtual environments. Social Influence, 4(3), 231-248. doi: $10.1080 / 15534510802643750$

Hall, E. T. (1963). A system for the notation of proxemic behavior. American Anthropologist, 65(5), 1003-1026. doi: 10.1525/aa.1963.65.5.02a00020

Hall, E. T. (1966) The hidden dimension. Anchor Books.

Hayduk, L. A. (1983). Personal space: Where we now stand. Psychological Bulletin, 94(2), 293. doi: 10.1037/0033-2909.94.2.293

Jones, S. E., \& Aiello, J. R. (1979). A test of the validity of projective and quasi-projective measures of interpersonal distance. Western Journal of Communication (includes Communication Reports), 43(2), 143-152. doi: 10.1080/10570317909373962

Lombard, M., \& Ditton, T. (1997) At the heart of it all: The concept of presence. Journal of Computer-Mediated Communication, 3(2). Retreived from http://www.ascusc.org/jcmc/vol3/issue2/lombard.html doi: 10.1111/j.1083-6101.1997.tb00072.x

McCall, C., Blascovich, J., Young, A., \& Persky, S. (2009). Proxemic behaviors as predictors of aggression towards Black (but not White) males in an immersive virtual environment. Social Influence, 4(2), 138-154. doi: 10.1080/15534510802517418

Menshikova, G. Y. (2013). An investigation of 3D images of the simultaneous-lightness-contrast illusion using a virtual-reality technique. Psychology in Russia: State of the Art, 6(3), 49-59. doi: 10.11621/pir.2013.0305

Menshikova, G. Y., Kovalev, A. I., Klimova, O. A., \& Chernorizov, A. M. (2015). Eye movements as indicators of vestibular dysfunction. Perception, 44(8-9), 1103-1109. doi: $10.1177 / 0301006615594916$

Oosterhout, T. \& Visser, A. (2008). A Visual Method for Robot Proxemics Measurements. 3rd ACM. In IEEE International Conference on Human-Robot Interaction Workshop on Metrics for Human-Robot Interaction, Technical Report 471, Amsterdam, Netherlands.

Oxford dictionary. (n.d.). Retrieved from www.oxforddictionaries.com/definition/ english/ avatar

Rosegrant, T. J., \& McCroskey, J. C. (1975). The effects of race and sex on proxemic behavior in an interview setting. Southern Journal of Communication, 40(4), 408-418. doi: $10.1080 / 10417947509372282$

Schegloff, E. A. (1998). Body torque. Social Research, 65(3), 535-596.

Watson, O. M. (1970). Proxemic behavior: A cross-cultural study. The Hague, The Netherlands: Mouton De Gruyter. doi: 10.1515/9783111323190

Willis, F. N. (1966). Initial speaking distance as a function of the speakers' relationship. Psychonomic Science, 5, 221-222. doi: 10.3758/BF03328362

Witmer, B. G. \& Singer, M. J. (1998) Measuring presence in virtual environments: A presence questionnaire. PRESENCE: Teleoperators and Virtual Environment, 7, 225-240. doi: $10.1162 / 105474698565686$ 
Zinchenko, Yu. P., Kovalev, A. I., Menshikova, G. Ya., \& Shaigerova, L. A. (2015). Postnonclassical methodology and application of virtual reality technologies in social research. Psychology in Russia: State of the Art. 8(4), 60-71. doi: 10.11621/pir.2015.0405

Zinchenko, Yu. P., Menshikova, G. Ya., Bayakovsky, Yu. M., Chernorizov, A. M., \& Voiskounsky, A. E. (2010). Technologies of virtual reality in the context of world-wide and Russian psychology: Methodology, comparison with traditional methods, achievements and perspectives. Psychology in Russia: State of the Art, 3, 12-45. doi: 10.11621/pir.2010.0001

Original manuscript received September 07, 2016 Revised manuscript accepted October 20, 2016

First published online December 15, 2017 